\title{
Development of a Desktop Universal Testing Machine with a Full-field Optical Extensometer
}

\author{
Wei-chen Lee ${ }^{1 *}$ and Frank Laufer ${ }^{2}$ \\ ${ }^{1}$ Department of Mechanical Engineering, National Taiwan University of Science and Technology, \\ Taipei 10607, Taiwan, Republic of China \\ ${ }^{2}$ Department of Mechanical Engineering, Hochschule Esslingen, \\ 73728 Esslingen, Germany
}

(Received February 28, 2019; accepted May 8, 2019)

Keywords: deformation, digital image correlation, universal testing machine

The objective of this research was to develop a 500-N desktop universal testing machine (UTM) with the capability to find the full-field deformation of a sheet specimen during tensile testing. The machine is equipped with a CMOS camera as the sensor to capture the images of the specimen during material tests. Using the open source 2D digital image correlation (DIC) program Ncorr, we can analyze the images and obtain the full-field deformation and strain. In this research, it was found that the speckle patterns generated by a spray paint and an airbrush were equivalently good. The deviation of the displacement measured by the DIC method with the two patterns can be less than $1 \%$. More tests show that the $2 / 3$-inch-diameter lens with a large field of view can have more accurate strains than the other 1-inch-diameter lens with a small field of view used in this research. The static and continuous loading material tests demonstrated that the strains measured on the UTM were consistent with those measured on a commercial system.

\section{Introduction}

For a reliable, cost-efficient, and sustainable design of components, accurate knowledge of the material behavior in the intended application is of particular importance. This knowledge is determined in material tests under different conditions and loads. While performing the material tests with a universal testing machine (UTM), the deformation of the material and applied force are measured to obtain the properties of the material. Furthermore, the measured values are required to derive several indirectly measurable quantities such as strain or stress. Several possible methods can be used to measure the deformation; these differ in the sensors for the deformation measurement. Which measuring method is most suitable must be considered on a case-by-case basis. Such methods are divided into the following categories:

- use of an extensometer (contact or noncontact) and

- use of a strain gauge.

Contact extensometers have physical contact with the surface of the specimen. Knife edges at the end of the two sensor arms generate a form-fit with the surface and move with ${ }^{*}$ Corresponding author: e-mail: wclee@mail.ntust.edu.tw https://doi.org/10.18494/SAM.2019.2368 
the deformation of the specimen. Inside each contact extensometer, the two sensor arms are fixed at a defined distance. When this distance changes or when the arms are deflected, a different electrical signal is detected in the range sensor. An industrial system is specified with a resolution of $0.1 \mu \mathrm{m}$, allowing even the slightest deformation to be measured. On the other hand, an extensometer of this type can only measure a few millimeters, which makes it unsuitable for detecting large deformations. Furthermore, it is prone to damage, especially in the moment of a fracture in a specimen. Another characteristic of this extensometer is the high repeatability of measurements. The weight of this extensometer may affect the measurement, especially for thin materials or materials with low stiffness such as foils or polymers.

Noncontact extensometers have no physical contact with the specimen, making them less prone to damage. One measurement method is to apply dots on the surface of the specimen and acquire continuous images of them by using a camera as the sensor as shown in Fig. 1. An algorithm identifies the dots and tracks their movement to determine the displacement. Both the longitudinal and transverse deformations can be detected. The dots on the surface are necessary for the unique identification of the movement and have to be applied before testing. Another noncontact measurement method is the use of a laser extensometer. This measurement method does not require the application of marks on the surface. A laser beam is pointed on the surface. Owing to the different surface structures in each point, the laser is reflected uniquely and detected by a sensor as a speckle pattern. Each mark on the surface is like a "fingerprint" and is detected at each step of deformation. While testing, these marks are tracked and enable the calculation of the strain. Noncontact measurement methods have the advantage of having no physical contact between the specimen and the measuring device. Therefore, no effect on the measurement occurs, which is especially advantageous for ductile or thin specimens.

Another measurement method is the use of a strain gauge as the sensor applied to the surface of the specimen. While testing, the deformations are measured directly and locally since the strain gauge is adhered to the surface of the specimen. Strain gauges are based on the principle of a changing electrical resistance of an elongated conductor. The most common type is a foil strain gauge made of a metal foil arranged as a grid on a plastic foil base. Strain gauge circuits, especially quarter and half bridges, are particularly sensitive to temperature changes, because the changes cause the strain gauge and leads to expansion or shrinkage, thus bringing the bridge

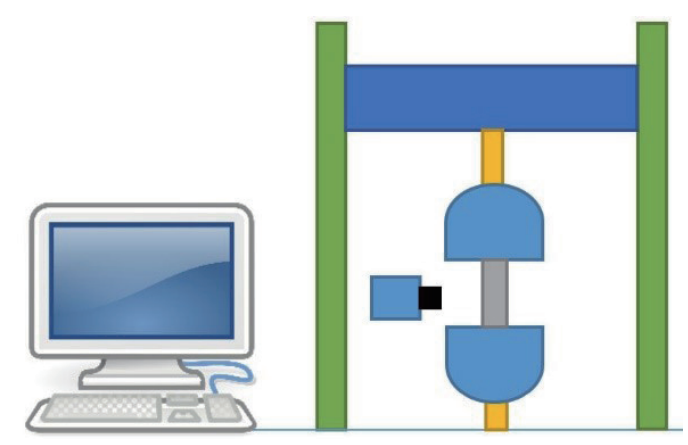

Fig. 1. (Color online) Schematic of the UTM with a camera as the sensor. 
out of balance. Another method to compensate for an uneven temperature effect on the leads is to twist them. Owing to the time-consuming application of gauges, these are not typically used in material tests but rather in the determination of loads on components in their applications.

The extensometers and strain gauges usually cannot show the full-field deformation or full-field strain, which is somehow less informative when performing material tests such as tensile, compressive, and bending tests. One method that can show the full-field deformation is the digital image correlation (DIC) method. Several researchers have studied how to apply 2D or 3D DIC methods to material testing ${ }^{(1-4)}$ or strain measurement. ${ }^{(5)}$ Because the full-field deformation can be viewed, the comparison can be easily made between the results of the DIC measurement and finite-element analysis. ${ }^{(6,7)}$ The DIC methods can also be integrated with high-speed photography to measure the deformation at a high strain rate. ${ }^{(8,9)}$ This technique can also be used to measure the material properties on the microscale. ${ }^{(10)}$ When performing material tests, it will be advantageous if DIC is available.

The objective of this research was to develop a 500-N desktop UTM equipped with a camera as the sensor to observe the full-field deformation of a sheet specimen during tensile testing by using an open source 2D DIC algorithm. This work can be beneficial to researchers who are interested in developing a UTM with a DIC capability, or to those who would like to add a DIC capability to their existing UTM.

\section{Materials and Methods}

The structure of the UTM that we developed and the open source DIC algorithm are described as follows.

\subsection{Hardware of the UTM}

The desktop UTM built for this research can apply a test force of $500 \mathrm{~N}$ on a sheet specimen. An overview of this UTM is shown in Fig. 2. The linear scale for position monitoring is attached onto the right side next to the frame. The linear scale is the Model WTA5 slimtype linear scale by CARMAR (Taiwan) with a measuring length of 0-200 mm. According to the manufacturer, the accuracy of the device is $\pm 6 \mu \mathrm{m}$. Moreover, two limit switches were implemented to limit the movement of the slide as protection against damage.

A camera for image acquisition is also part of the hardware. In this UTM, the Basler agA2500-14mg, a camera for industrial applications, was utilized. This camera is equipped with a $5.7 \mathrm{~mm}(h) \times 4.3 \mathrm{~mm}(v)$ CMOS sensor with a $2592 \times 1944$ pixel resolution, which is 5 MPs in total. Owing to these dimensions, the pixel size is $2.2 \times 2.2 \mu \mathrm{m}^{2}$. For this camera, the following two lenses were used to compare their results:

- The Computar M1614-MP2 has a diameter of 2/3 inch $(17 \mathrm{~mm})$ and a focal length of $f=16 \mathrm{~mm}$. The ratio between the focal length and the aperture diameter is maximal at $\mathrm{F}$ 1.4. Both focus and aperture are manually adjusted for this lens.

- A lens with a diameter of 1 inch $(25.4 \mathrm{~mm}), f=12 \mathrm{~mm}$, and a maximal ratio of $\mathrm{F} 1.8$ is also manually adjustable. 


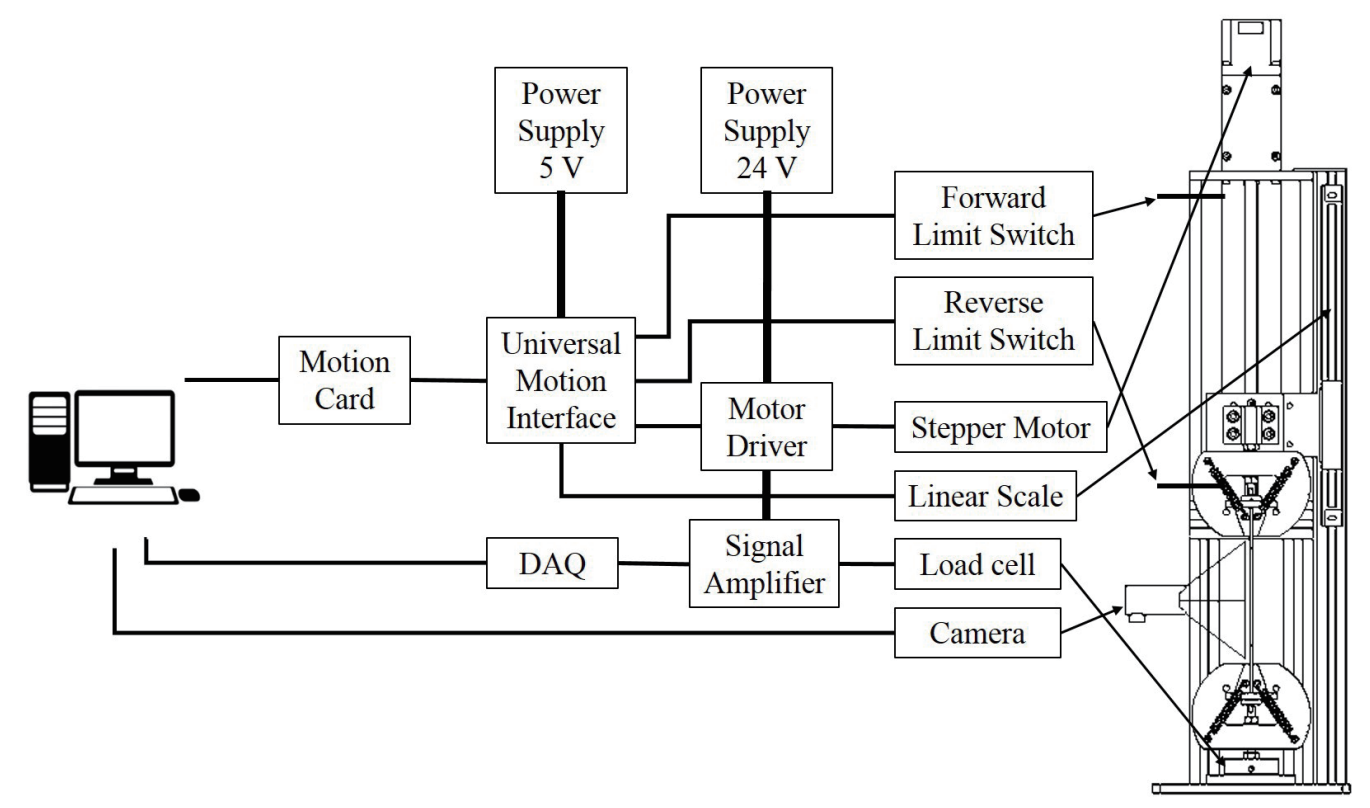

Fig. 2. Overview of the hardware for the UTM.

A stepper motor is mounted on the top of the machine to provide the motion of the upper wedge grip. The motor is a hybrid stepper Model CKM266-NAC (Chyen Den, Taiwan). The motor exerts a holding torque of $12 \mathrm{~kg}-\mathrm{cm}$ or $1.18 \mathrm{~N}-\mathrm{m}$, which was tested to be large enough to generate a $500 \mathrm{~N}$ pull force through a trapezoidal screw.

A load cell, which is at the bottom of the lower wedge grip, is a device used to measure force. It is based on measuring the elastic deformation of a spring element resulting from the force to be measured with the aid of strain gauges inside. For the research, the load cell Model 8524 by Burster was used. According to the manufacturer, the accuracy of this device is $\pm 0.25 \%$. This sensor is designed to measure unidirectional tension or compression forces up to $500 \mathrm{~N}$. In the load cell, four strain gauges are applied, and this forms a full bridge. The recommended excitation voltage for the bridge is $5 \mathrm{~V}$, which leads to an output signal in the range of $7.5 \mathrm{mV}$. This signal is too small to be measured directly; therefore, an amplifier is added after the load cell to raise this signal to a level that makes it usable. An amplifier Model CPJ by SCAIME is suitable for performing this operation. This device amplifies the input signal from the load cell up to $\pm 10 \mathrm{~V}$ and the current up to $20 \mathrm{~mA}$.

After amplification, a signal is acquired and transmitted to the software to be converted to a strain and subsequently to a force. For data acquisition, a myDAQ by National Instruments was used. This compact USB device is equipped with two analog inputs (AIs) and outputs (AOs) as well as a digital programmable function interface, which can be configured as input or output. One of the AIs is used to acquire the signal of the load cell. We used the software LabVIEW also by National Instruments to process the signals acquired by myDAQ. In LabVIEW, a handy function for myDAQ is available to input the signal to the software.

Owing to the compatibility of the software and hardware components, LabVIEW is also used to program the machine controls. Three control or acquisition branches, which are the load 
cell, stepper motor, and linear scale branches, are executed simultaneously in the LabVIEW environment, and signals are transferred between them. Figure 3 shows a simplified flow chart of the machine control.

\subsection{DIC method}

The idea behind DIC is to record digital images containing measurement data and to employ algorithms to analyze these images for the extraction of the measurement data. In recent years, the use of this method in engineering has increased significantly. DIC is a method for the noncontact optical measurement of displacements with the automatic image analysis of previously acquired images made by matching them and finding the correlation between certain areas to create displacement vectors. In engineering applications, often minuscule motion is of interest, i.e., measuring deformations in material testing. Therefore, the requirements in the resolution are high. ${ }^{(11)}$

In an image, many pixels have the same grayscale value. Therefore, no unique correlation between the pixels in the two images is possible. To create a correspondence, the pixels have to be unique. To achieve this, a pattern is applied to the surface of a specimen. However, when a repeating pattern is used, the problem described above arises again. A grid, for example, has several identical points and, therefore, no unique correlations are created. This uniqueness is achieved by applying a speckle pattern on the surface. A speckle pattern is a randomly distributed, ideally isotropic pattern with random particle shapes. The key factor of this pattern is the high information content, which enables a unique identification all over the surface via a comparison of certain regions. Several papers show how important the quality of this pattern is for achieving reliable results. ${ }^{(12-16)}$ Those researchers conducted research that focused on evaluating the quality of a speckle pattern. Further research is being done to continuously improve the pattern. ${ }^{(17)}$

In addition to pattern quality, other factors affect the DIC measurement method. Changing light conditions between the images leads to a change in grayscale value, and thus affecting the identification of the pixels. An algorithm that is less sensitive to changing light conditions was developed. ${ }^{(18)}$

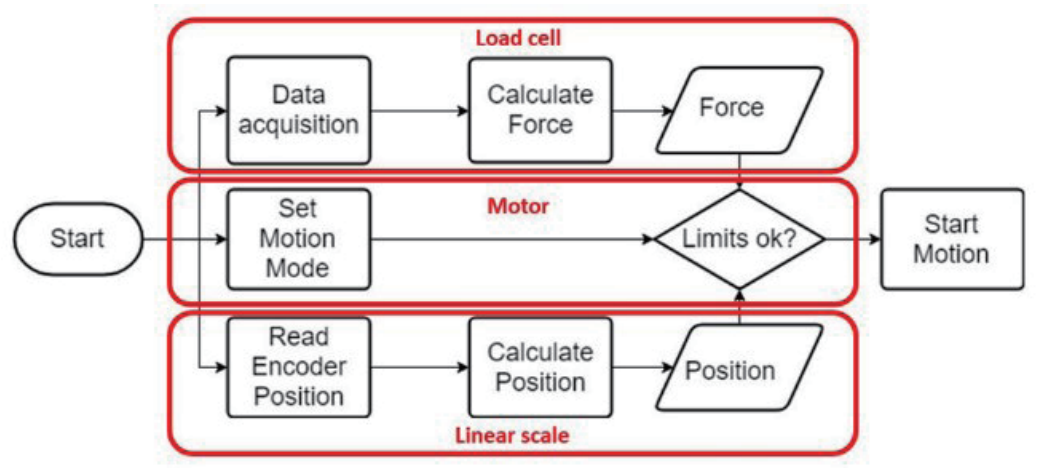

Fig. 3. (Color online) Flow chart of the machine control with three simultaneously executed branches. 
For the procedure discussed, a model that allows the detection of motion in successive images in mathematical terms must be created. Therefore, the method of template matching is performed. It is based on the minimization of the greyscale value difference between two subsets from a reference image (template) and a deformed image. A subset is a small area of the surface of the specimen defined in an image. However, this method leads to a decrease in correspondence with the increase in the deformation of the subsets, termed decorrelation. For this reason, a shape function is employed to take this deformation into account. ${ }^{(11)}$

Various noncommercial DIC software programs are available for optical strain measurement. Furthermore, commercial software programs such as VIC, GOM Correlate, and StrainMaster are available. The open source DIC software Ncorr was adopted in this research. It is a 2D DIC software developed at the Georgia Institute of Technology. ${ }^{(19)}$ In validations, the software shows good results compared with commercial DIC software for the measurement of deformations of metals and polymers. ${ }^{(20)}$ The algorithm was written in $\mathrm{C}++$, and the user interface was written in MATLAB. This provides a clear interface and easy handling of the software. For more specific applications, the developers allow the user free access to the source code to adjust it for their applications.

\subsection{Specimen preparation}

The specimens used in this research were flat aluminum specimens cut using a waterjet from a sheet. Each specimen was made according to ASTM E8 with a thickness of $0.5 \mathrm{~mm}$ and a width of $12.5 \mathrm{~mm}$. Afterward, the speckle pattern was applied to the specimen. As mentioned previously, the pattern has a significant effect on the results. Therefore, three different patterns were applied and subsequently evaluated to find the pattern that produced the best results with the DIC measurement.

A simple method of generating a pattern was to use a marker with a tip diameter of $0.5 \mathrm{~mm}$ and create the pattern by hand on the surface of the specimen. The second way to apply a pattern was to use a standard can of spray paint. A continuous horizontal motion at about $0.5 \mathrm{~m}$ from the nozzle to the surface leads to an even and isotropic pattern. For this method, there is a risk of paint splashes, which must be avoided to obtain an even particle size.

The third way to apply a pattern was to use the spray paint from the can and spray it via an airbrush pistol. This method created the finest pattern due to the small nozzle diameter of $0.3 \mathrm{~mm}$. The air pressure has a significant effect. A high pressure leads to finer atomization of the paint and reduces the risk of clogs in the nozzle. On the other hand, the small drops of paint dry quickly and may be dry before they reach the surface. Spray tests showed that a three bar pressure generated good results. Figure 4 shows the outcome of the three different patterns on the specimens. It can be observed that the speckles were getting finer from the top pattern (made by the marker) to the bottom pattern (made by the airbrush).

In Fig. 5, the three patterns are shown in a closer view. A microscope with $20 \times$ and $45 \times$ magnifications was used to examine the pattern more closely. The diameter of the dots made with a marker is about $0.5 \mathrm{~mm}$, the spray paint produces particles of different sizes and shapes, and the airbrush produces the finest particles. 


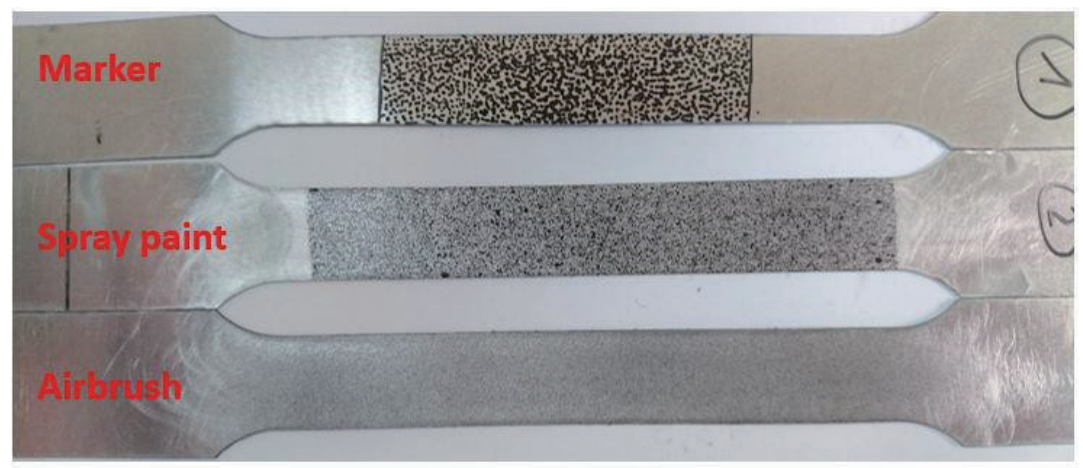

Fig. 4. (Color online) Patterns created with marker by hand, with spray paint and with airbrush.
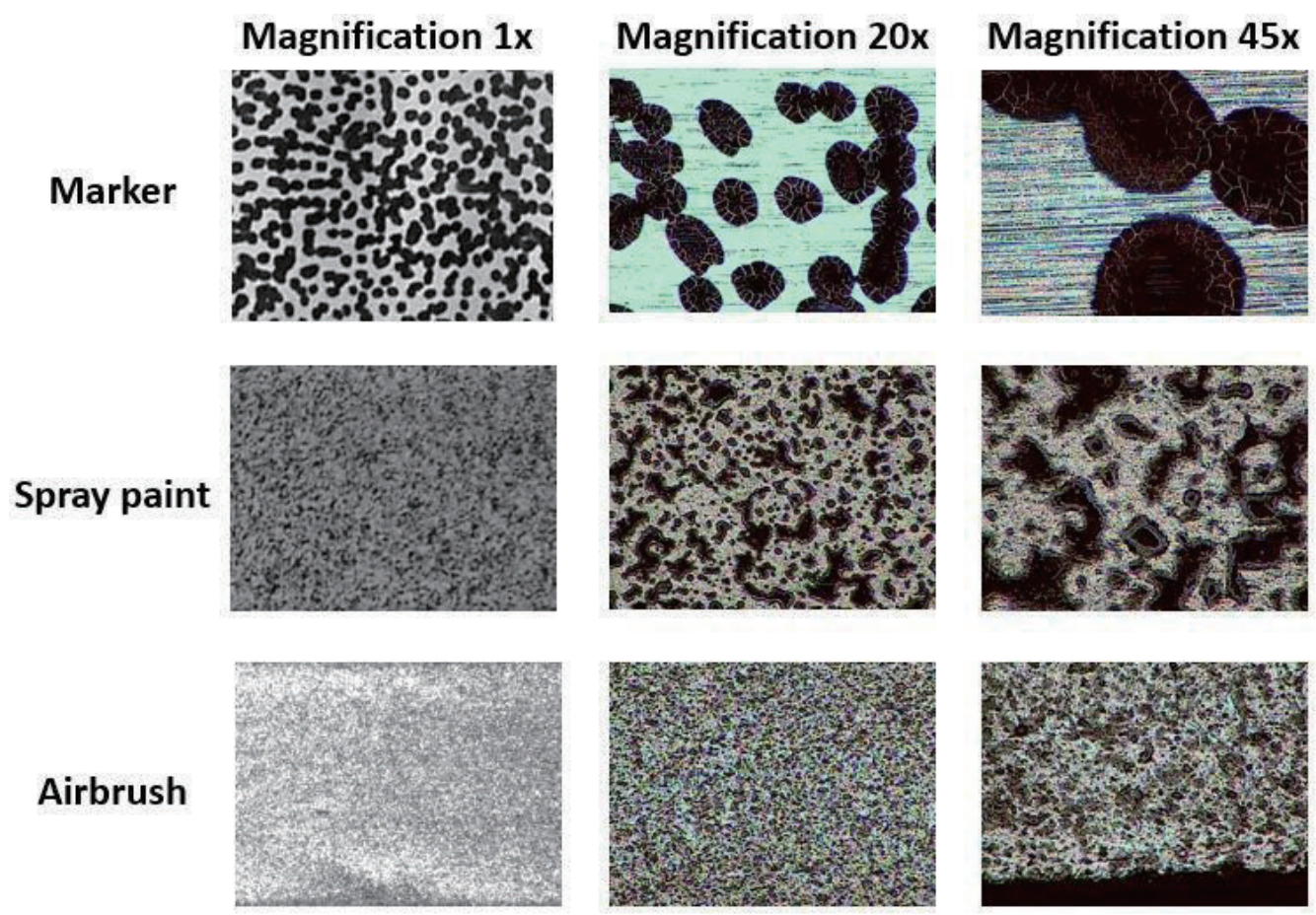

Fig. 5. (Color online) Patterns created with different methods under microscope magnification.

\section{Results and Discussion}

\subsection{Pattern tests on the UTM}

Before performing strain measurement tests, the accuracy of the Basler camera with the two lenses was tested by comparing the measured displacement of the force-free specimen using a linear scale with the results of the DIC software. During the test, the specimen was only clamped in the upper wedge grip to obtain a constant displacement with no strain. Since the specimen was not loaded, no deformation of the machine occurs and the value of the linear 
scale is used as a reference value. Three different patterns were investigated to find the optimal pattern for further strain measurement. In Fig. 6, the experimental setup is shown. The camera with a Computar lens diameter of $2 / 3$ inch was used to capture the images of the specimen with the aid of the LED light source. In Table 1 , an overview of the deviations in median values to the linear scale and range of displacement is shown. A displacement of $2 \mathrm{~mm}$ was measured using the linear scale. The deviation of the median value with the spray paint pattern was $0.009 \mathrm{~mm}$, which was less than $0.5 \%$ of the total displacement. For the test with the airbrush pattern, the deviation of $0.016 \mathrm{~mm}$ also showed good accuracy. In these tests, the marker pattern showed a deviation of $-0.051 \mathrm{~mm}$ or $-2.5 \%$. These results, especially the comparison between the marker and the other two patterns, have been partly confirmed, since it was mentioned that smaller particles of the pattern lead to a higher accuracy when the smaller subset in DIC was used. ${ }^{(21)}$ The radius of the subset used here was 65 pixels. Because the difference between the spray paint result and the airbrush result was minimal, both should be good to be used. Since the spray paint pattern leads to the highest accuracy, it was used for the following tests.

\subsection{Lens tests on the commercial system}

The next step was to clamp both ends of the specimen and perform tests to measure strains. For the validation of the results acquired with our UTM, more results from a validated testing machine are required. Therefore, tensile tests on the commercial system, Instron 3365 (Instron, USA), were performed. Because the capacity of the commercial system is $5000 \mathrm{~N}$, which is much larger than the $500 \mathrm{~N}$ we used in our tests, these tests provided results on the machine with sufficient stiffness. The procedure of the tests was with successive increases in force in certain steps until $500 \mathrm{~N}$ was reached. The test setup is shown in Fig. 7. The same DIC program, Ncorr, was used for strain measurement on both our UTM and the commercial system.

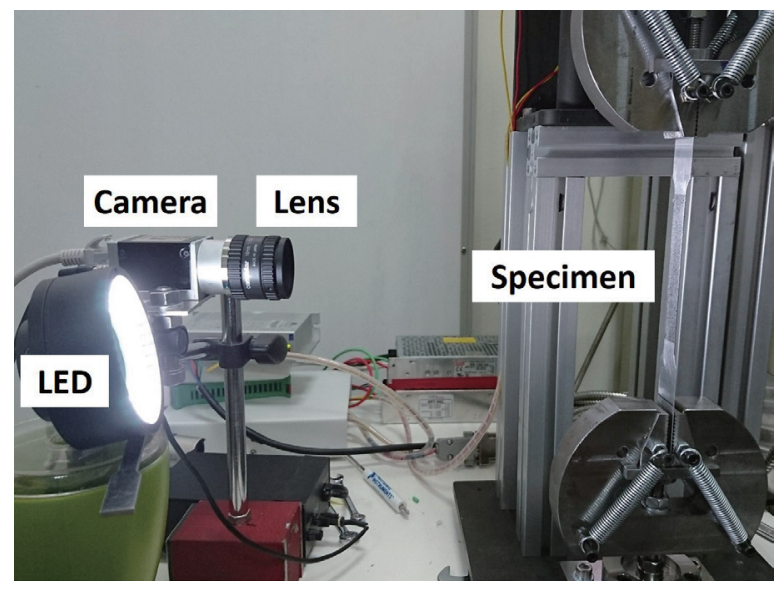

Table 1

Test results on three different patterns using the Basler camera with a computar lens of $2 / 3$ inch diameter.

\begin{tabular}{lccc}
\hline & \multicolumn{3}{c}{ Displacement $(\mathrm{mm})$} \\
\cline { 2 - 4 } & Min. & Med. & Max. \\
\hline Linear scale & & 2.000 & \\
Marker & 1.941 & 1.949 & 1.957 \\
Spray paint & 2.001 & 2.009 & 2.017 \\
Airbrush & 2.008 & 2.016 & 2.025 \\
\hline
\end{tabular}

Fig. 6. (Color online) Experimental setup with the Basler camera and the LED light for the pattern tests. 
Two different lenses are tested here to find out which lens leads to more accurate results. Images were taken with the 2/3-inch-diameter lens at a distance of $260 \mathrm{~mm}$ from the specimen. The lens with a 1 inch diameter had a smaller focal length, and the camera was placed $40 \mathrm{~mm}$ from the specimen. Figure 8 shows the images taken with the two lenses. It can be seen that the field of view of the 1 -inch-diameter lens was much smaller than that of the $2 / 3$-inchdiameter lens.

An overview of the test results is shown in Table 2. The results for images taken with the 1-inch-diameter lens showed that a median value in the $Y$-direction (longitudinal direction) was $2.77 \times 10^{-3}$, which was about twice as high as that obtained by using the 2/3-inch-diameter lens or strain gauge. According to these investigations, the images taken with the 1-inch-diameter lens showed that the values were very high with a wide range compared with the images taken with the 2/3-inch-diameter lens. The reason for this is the fact that the 1-inch-diameter lens with a smaller field of view is more sensitive to the camera noise or ambient light. Then, it was determined that the 1-inch-diameter lens was not suitable for measuring the strain; thus, it was not used in further tests.

\subsection{Strain measurement on the UTM and commercial system}

To validate the test results from the UTM that we developed, the strain measurement results were compared with the results measured with a strain gauge and those measured with the

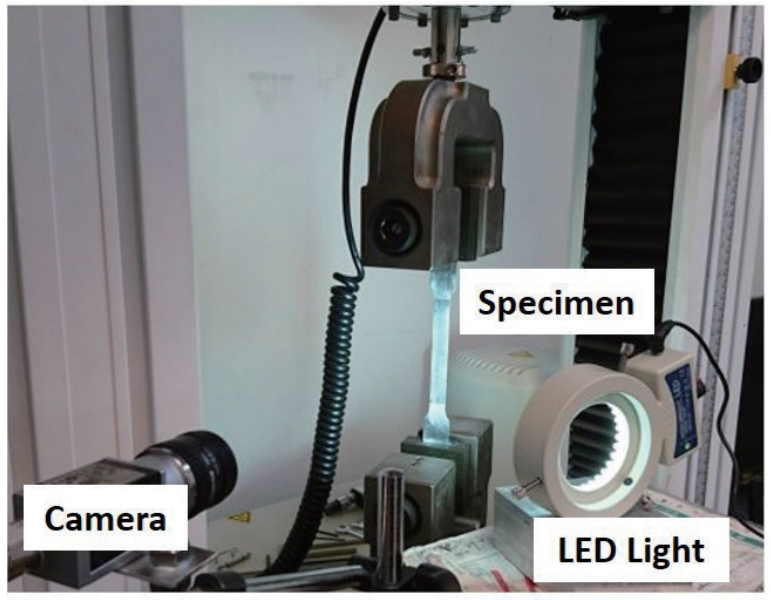

Fig. 7. (Color online) Test setup for validation tests on Instron 3365.

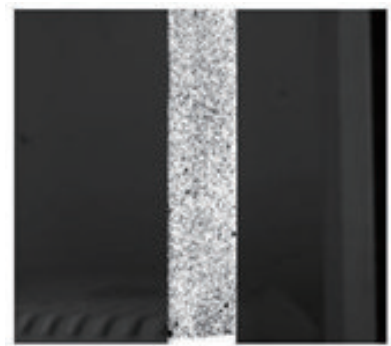

2/3" lens

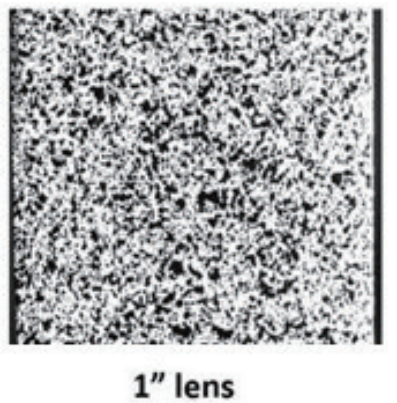

1" lens

Fig. 8. Images captured by using the lenses with diameters of $2 / 3$ and 1 inch.

Table 2

Test results for $Y$-strains with different lenses on the commercial system (Instron 3365) using DIC.

\begin{tabular}{|c|c|c|c|}
\hline & \multicolumn{3}{|c|}{$Y$-strain $\left(\times 10^{-3}\right)$} \\
\hline & Min. & Med. & Max \\
\hline Strain gauge & & 1.30 & \\
\hline Commercial, spray paint, 2/3-inch-diameter lens & 1.20 & 1.46 & 1.80 \\
\hline Commercial, spray paint, 1-inch-diameter lens & 1.36 & 2.77 & 3.57 \\
\hline
\end{tabular}


commercial system Instron 3365. Table 3 shows a summary of the results at a test force of $500 \mathrm{~N}$. The same DIC program Ncorr was used for analyzing the images captured on our UTM as well as on the commercial system.

For the $Y$-strain results from the commercial system, the median values from the spray paint pattern with the 2/3-inch-diameter lens are close to the strain gauge results. A deviation of $1.6 \times 10^{-4}$ occurs. Besides the good results measured by the commercial system, the results from the test with the UTM and 2/3-inch-diameter lens show a slightly larger deviation, $3.3 \times 10^{-4}$, and a smaller range of distribution comparable to the results from the commercial system. The tests on the UTM with the spray paint pattern and 2/3-inch-diameter lens also prove to be consistent for the full-field measurement of strains comparable to the measurement results on the commercial system.

All of the previous results were compared and validated at a static point of $500 \mathrm{~N}$. To simulate an increasing test force and validate the accuracy of the DIC measurement over the full test force range, tests with a successively increasing force were performed. For this test, only the specimen with the spray paint pattern was used on the basis of previous investigations. Tests were performed with identical hardware and DIC software on the commercial system and UTM. To validate the values, the strain gauge results were used again. As in the previous static test, the $Y$-strain was subject to investigation to determine the full-field strains. The full test force range is shown in Fig. 9. Over the full test force range, the deviation in the DIC results on

Table 3

Test results for $Y$-strains on the commercial system (Instron 3365) and our UTM using DIC.

\begin{tabular}{|c|c|c|c|}
\hline & \multicolumn{3}{|c|}{$Y$-strain $\left(\times 10^{-3}\right)$} \\
\hline & Min. & Med. & Max. \\
\hline Strain gauge & & 1.30 & \\
\hline Commercial, spray paint, 2/3-inch-diameter lens & 1.20 & 1.46 & 1.80 \\
\hline UTM, spray paint, 2/3-inch-diameter lens & 1.36 & 1.63 & 1.92 \\
\hline
\end{tabular}

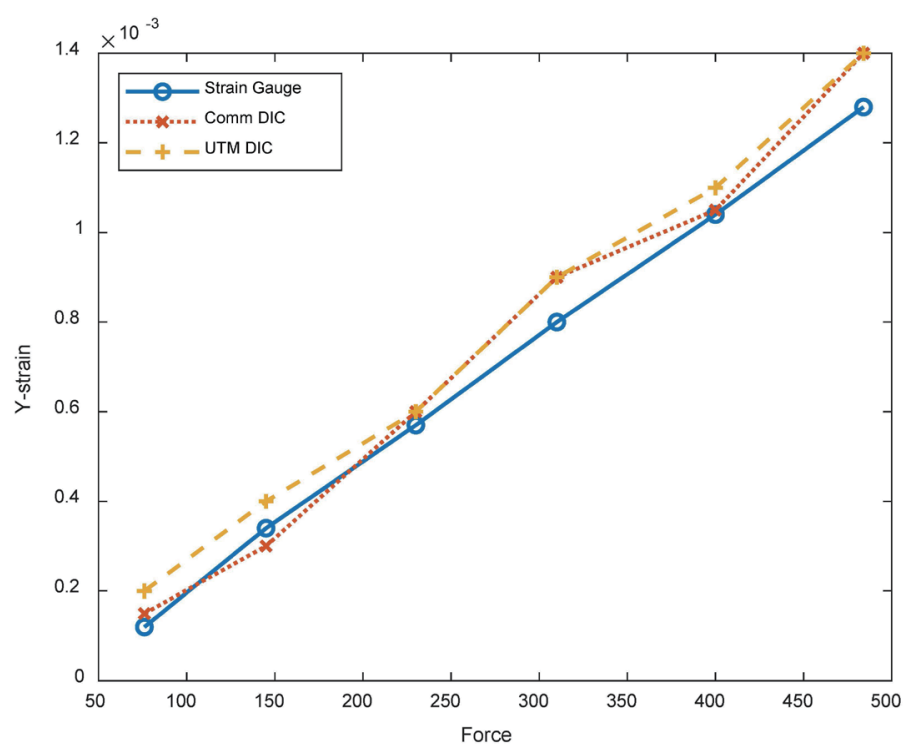

Fig. 9. (Color online) Comparison of $Y$-strain values under increasing test force up to $484 \mathrm{~N}$. 
the commercial system from the strain gauge is small with a maximum of $1.2 \times 10^{-4}$. Almost identical were the results of the DIC measurements on the UTM with a maximum deviation of $1.2 \times 10^{-4}$ at the maximum test force of $484 \mathrm{~N}$. The validation showed good accuracy of the DIC measurements compared with the strain gauge measurements. Furthermore, the results of our UTM are comparable to the results of the commercial system.

\section{Conclusions}

A UTM was built for this research to realize a UTM with a DIC capability with the use of a CMOS sensor. The open source DIC software Ncorr was adopted for this UTM. Several material tests were performed to find out the best conditions for conducting tests on the UTM to obtain accurate results, and the important results are summarized as follows.

- Three different methods of producing a speckle pattern were tested. Measurements with the spray paint pattern produced accurate results comparable to the airbrush pattern. The results of the marker pattern made by hand were less accurate than those of the spray paint and airbrush patterns. The deviations of the spray and airbrush patterns in the displacement tests were less than $1 \%$ from the displacement measured on the linear scale, and that of the marker pattern was about $2.5 \%$.

- The 2/3-inch-diameter lens with a large field of view generated more accurate results than the 1-inch-diameter lens with a small field of view. The deviation of the strain measured by using the 1-inch-diameter lens was about twice as high as that obtained by using the 2/3-inch-diameter lens.

- The strains measured by the UTM developed in this research and those measured by the commercial system were consistent. For the static loading at $500 \mathrm{~N}$, the strains measured by our UTM and the commercial system were about $3.3 \times 10^{-4}$ and $1.6 \times 10^{-4}$ more than that measured by using the strain gauge, respectively. For the continuous loading, the results measured by our UTM, the commercial system Instron 3365, and the strain gauge still showed good consistency.

This paper can serve as reference for those who would like to build a UTM with a DIC capability. Further improvement of the UTM structure to make it more robust will be a future work to increase its accuracy.

\section{Acknowledgments}

This work was financially supported by the "Center for Cyber-physical System Innovation" from The Featured Areas Research Center Program within the framework of the Higher Education Sprout Project by the Ministry of Education (MOE) in Taiwan.

\section{References}

1 F. Zhu, P. Bai, J. Zhang, D. Lei, and X. He: Opt. Laser Eng. 65 (2014) 81. http://doi.org/10.1016/ j.optlaseng.2014.06.013

2 C. P. Dick and Y. P. Korkolis: Int. J. Solids Struct. 51 (2014) 3042. https://doi.org/10.1016/j.ijsolstr.2014.04.023 
3 J. T. Cantrell, S. Rohde, D. Damiani, R. Gurnani, L. DiSandro, J. Anton, A. Young, A. Jerez, D. Steinbach, C. Kroese, and P. G. Ifju: Rapid Prototyping J. 23 (2017) 811. https://doi.org/10.1108/RPJ-03-2016-0042

4 J. Na, W. Mu, G. Qin, W. Tan, and L. Pu: Int. J. Adhes. Adhes. 85 (2018) 138. https://doi.org/10.1016/ j.ijadhadh.2018.05.027

5 N. L. Clelland, B. Yilmaz, and J. D. Seidt: Clin. Implant Dent. Related Res. 15 (2013) 271. https://doi. org/10.1111/j.1708-8208.2011.00411.x

6 J. Qin, R. Chen, X. Wen, Y. Lin, M. Liang, and F. Lu: Mater. Sci. Eng. A 586 (2013) 62. https://doi.org/10.1016/ j.msea.2013.07.091

7 S. Duan, F. Mo, X. Yang, Y. Tao, D. Wu, and Y. Peng: Compos. Part B-Eng. 88 (2016) 101. https://doi. org/10.1016/j.compositesb.2015.11.006

8 Y. Liu, D. Dong, L. Wang, X. Chu, P. Wang, and M. Jin: Mater. Sci. Eng. A 627 (2015) 296. https://doi. org/10.1016/j.msea.2014.12.103

9 L. Li, W. G. Guo, X. Yu, and D. X. Fu: Ceram. Int. 43 (2017) 6684. https://doi.org/10.1016/j.ceramint.2017.02.064

10 L. Banks-Sills, J. Shklovsky, S. Krylov, H. A. Bruck, V. Fourman, R. Eliasi, and D. Ashkenazi: Strain 47 (2011) 288. https://doi.org/10.1111/j.1475-1305.2009.00692.x

11 H. Schreier, J. J. Orteu, and M. A. Sutton: Image Correlation for Shape, Motion and Deformation Measurements: Basic Concepts, Theory and Applications (Springer, New York, 2009) p. 1. https://doi. org/10.1007/978-0-387-78747-3

12 J. Zhang, A. Sweedy, F. Gitzhofer, and G. Baroud: Opt. Laser Eng. 100 (2018) 259. https://doi.org/https://doi. org/10.1016/j.optlaseng.2017.09.012

13 G. Crammond, S. W. Boyd, and J. M. Dulieu-Barton: Opt. Laser Eng. 51 (2013) 1368. https://doi.org/https:// doi.org/10.1016/j.optlaseng.2013.03.014

14 J. Park, S. Yoon, T.-H. Kwon, and K. Park: Opt. Laser Eng. 91 (2017) 62. https://doi.org/https://doi.org/10.1016/ j.optlaseng.2016.11.001

15 B. Pan, Z. Lu, and H. Xie: Opt. Laser Eng. 48 (2010) 469. https://doi.org/https://doi.org/10.1016/ j.optlaseng.2009.08.010

16 T. Hua, H. Xie, S. Wang, Z. Hu, P. Chen, and Q. Zhang: Opt. Laser Technol. 43 (2011) 9. https://doi.org/https:// doi.org/10.1016/j.optlastec.2010.04.010

17 G. F. Bomarito, J. D. Hochhalter, T. J. Ruggles, and A. H. Cannon: Opt. Laser Eng. 91 (2017) 73. https://doi. org/https://doi.org/10.1016/j.optlaseng.2016.11.005

18 B. Pan, D. Wu, and Y. Xia: Opt. Laser Eng. 44 (2012) 204. https://doi.org/https://doi.org/10.1016/ j.optlastec.2011.06.019

19 J. Blaber, B. Adair, and A. Antoniou: Exp. Mech. 55 (2015) 1105. https://doi.org/10.1007/s11340-015-0009-1

20 R. Harilal and M. Ramji: Proc. 9th Int. Symp. on Advanced Science and Technology in Experimental Mechanics (2014). https://doi.org/10.13140/2.1.4994.1442

21 D. Lecompte, A. Smits, S. Bossuyt, H. Sol, J. Vantomme, D. Van Hemelrijck, and A. M. Habraken: Opt. Laser Eng. 44 (2006) 1132. https://doi.org/https://doi.org/10.1016/j.optlaseng.2005.10.004 